in vivo $35: 131-140(2021)$

doi:10.21873/invivo.12240

\title{
Low DUSP4 Expression Is Associated With Aggressive Phenotypes and Poor Prognosis in Gastric Cancer
}

\author{
SEONGSIK BANG ${ }^{1}$, SEUNGYUN JEE ${ }^{1}$, HYUNSUNG KIM ${ }^{1}$, KISEOK JANG ${ }^{1}$, HOSUB PARK $^{1}$, \\ JAE KYUNG MYUNG ${ }^{1}$, DONGHO CHOI ${ }^{2}$, SU-JIN SHIN ${ }^{3}$ and SEUNG SAM PAIK ${ }^{1}$ \\ ${ }^{1}$ Department of Pathology, Seoul Hospital, Hanyang University College of Medicine, Seoul, Republic of Korea; \\ ${ }^{2}$ Department of Surgery, Seoul Hospital, Hanyang University College of Medicine, Seoul, Republic of Korea; \\ ${ }^{3}$ Department of Pathology, Gangnam Severance Hospital, \\ Yonsei University College of Medicine, Seoul, Republic of Korea
}

\begin{abstract}
Background/Aim: Dual-specificity protein phosphatase 4 (DUSP4) negatively regulates MAPK signaling and is involved in various cellular processes. We herein evaluated the relationship between DUSP4 expression and clinicopathological characteristics in a large series of gastric cancer samples. Materials and Methods: DUSP4 expression was examined by immunohistochemistry in 508 gastric cancer samples. Cases were classified according to the TCGA molecular classification and HER2 amplification. KaplanMeier plots were used to predict the relationship between mRNA expression of DUSP4 and survival. Results: Low expression of DUSP4 was significantly correlated with larger tumor size, higher pT category, positive nodal status, higher stage, lymphovascular invasion, perineural invasion, worse overall survival, and worse recurrence-free survival. No correlation was observed between DUSP4 expression and molecular characteristics. Bioinformatics analysis showed that low mRNA expression was associated with a poor prognosis. Conclusion: Low expression of DUSP4 is associated with aggressive phenotypes of gastric cancer and a poor prognosis.
\end{abstract}

Gastric cancer is a common epithelial malignant neoplasm of the digestive system. According to the GLOBOCAN 2018 database, gastric cancer shows the fifth highest incidence and the third highest mortality rate worldwide (1). It is the most common cancer in East Asia, including Korea. Although the

This article is freely accessible online.

Correspondence to: Seung Sam Paik, MD, Ph.D., Department of Pathology, Seoul Hospital, Hanyang University College of Medicine, 222 Wangsimni-ro, Seongdong-gu, Seoul, 04763, Republic of Korea. Tel: +82 222208960, e-mail: sspaik@ hanyang.ac.kr

Key Words: Gastric cancer, DUSP4, dual-specificity protein phosphatase 4 , prognosis. age-standardized rate of incidence and death is declining, it still has a high mortality rate (2). Therefore, it is important to investigate novel treatment and prognostic biomarkers for gastric cancer.

Surgical resection is the only method for curative treatment of gastric cancer, but the prognosis is poor for advanced or metastatic gastric cancer. Therefore, adjuvant chemotherapy has been performed to improve survival, and recent advances in molecular biology have led to discovery of various targeted therapies. For example, the use of trastuzumab, an anti-human epidermal growth factor receptor-2 (HER2) drug, was approved with cytotoxic drugs for the treatment of metastatic HER2-overexpressing gastric cancer. Additionally, clinical trials for other molecular targets (EGFR, CLDN18.2, VEGFR) are ongoing (3).

Dual-specificity protein phosphatase 4 (DUSP4), also known as mitogen-activated protein kinase phosphatase (MKP)-2, negatively regulates mitogen-activated protein kinase (MAPK) signaling and is involved in various cellular processes such as cell proliferation and immune response (4). There have been many studies on the role of DUSP4 in cancer. In vitro studies of EGFR-mutated lung cancer (5), papillary thyroid carcinoma $(6,7)$, and pancreatic cancer $(8)$ revealed increased DUSP4 expression. Low expression of DUSP4 was associated with a poor prognosis in a study of diffuse large B-cell lymphoma (9), whereas high expression of DUSP4 was associated with lymph node metastasis and extrathyroidal extension in a study of papillary thyroid carcinoma (7). The relationship between DUSP4 expression and aggressive features showed contradictory results in studies of colorectal cancer $(10,11)$. Low DUSP4 expression was associated with a poor prognosis in a study of triplenegative breast cancer (12); however, high DUSP4 expression was associated with a poor prognosis in early T-stage breast cancer (13). In vitro studies of gastric cancer cell lines revealed that up-regulated DUSP4 expression was associated with drug resistance, epithelial-mesenchymal transition, 
Table I. Clinicopathological characteristics of gastric cancer cases $(n=508)$.

\begin{tabular}{|c|c|c|c|}
\hline Characteristics & Case No. $(\%)$ & Characteristics & Case No. $(\%)$ \\
\hline Age, median (range, year) & $62(25-90)$ & Lauren classification & \\
\hline Gender & & Intestinal & $212(41.7 \%)$ \\
\hline Male & $352(69.3 \%)$ & Diffuse & $128(25.2 \%)$ \\
\hline Female & $156(30.7 \%)$ & Mixed (including indeterminate) & $168(33.1 \%)$ \\
\hline Tumor size, mean (range, $\mathrm{cm}$ ) & $4.1(0.3-20.0)$ & Lymphovascular invasion & \\
\hline Location (center of tumor) & & Present & $254(50.0 \%)$ \\
\hline Cardia & $15(3.0 \%)$ & Not identified & $254(50.0 \%)$ \\
\hline Fundus & $2(0.4 \%)$ & Perineural invasion & \\
\hline Body & $164(32.3 \%)$ & Present & $193(38.0 \%)$ \\
\hline Angle & $23(4.5 \%)$ & Not identified & $315(62.0 \%)$ \\
\hline Antrum & $296(58.3 \%)$ & pT category & \\
\hline Pylorus & $8(1.6 \%)$ & $1 \mathrm{a}$ & $167(32.9 \%)$ \\
\hline Gross type (Early gastric cancer) & & $1 \mathrm{~b}$ & $100(19.7 \%)$ \\
\hline Type I & $14(5.2 \%)$ & 2 & $48(9.4 \%)$ \\
\hline Type IIa & $26(9.7 \%)$ & 3 & $102(20.1 \%)$ \\
\hline Type IIb & $36(13.5 \%)$ & $4 a$ & $84(16.5 \%)$ \\
\hline Type IIc & $148(55.4 \%)$ & $4 \mathrm{~b}$ & $7(1.4 \%)$ \\
\hline Type III & $13(4.9 \%)$ & $\mathrm{pN}$ category & \\
\hline Mixed & $30(11.2 \%)$ & 0 & $299(58.9 \%)$ \\
\hline Borrmann type (Advanced gastric cancer) & & 1 & $55(10.8 \%)$ \\
\hline Borrmann type 1 & $5(2.1 \%)$ & 2 & $59(11.6 \%)$ \\
\hline Borrmann type 2 & $52(21.6 \%)$ & $3 a$ & $46(9.1 \%)$ \\
\hline Borrmann type 3 & $155(64.3 \%)$ & $3 b$ & $49(9.6 \%)$ \\
\hline Borrmann type 4 & $29(12.0 \%)$ & Stage (AJCC $8^{\text {th }}$ edition) & \\
\hline Histologic type (by WHO classification, 2019) & & IA & $240(47.2 \%)$ \\
\hline Tubular adenocarcinoma, well differentiated & $74(14.6 \%)$ & IB & $41(8.1 \%)$ \\
\hline Tubular adenocarcinoma, moderately differentiated & $123(24.2 \%)$ & IIA & $51(10.0 \%)$ \\
\hline Tubular adenocarcinoma, poorly differentiated & $121(23.8 \%)$ & IIB & $33(6.5 \%)$ \\
\hline Papillary adenocarcinoma & $2(0.4 \%)$ & IIIA & $50(9.8 \%)$ \\
\hline Mucinous adenocarcinoma & $14(2.8 \%)$ & IIIB & $44(8.7 \%)$ \\
\hline \multirow{2}{*}{$\begin{array}{l}\text { Poorly cohesive carcinoma (including signet ring } \\
\text { cell carcinoma) }\end{array}$} & & IIIC & $49(9.6 \%)$ \\
\hline & $100(19.7 \%)$ & Treatment & \\
\hline Other histologic subtypes* & $20(3.9 \%)$ & Surgery+adjuvant chemotherapy & $233(45.9 \%)$ \\
\hline Mixed adenocarcinoma & $54(10.6 \%)$ & Surgery & $275(54.1 \%)$ \\
\hline
\end{tabular}

*Other histological subtypes, adenosquamous carcinoma, squamous cell carcinoma, carcinoma with lymphoid stroma, hepatoid adenocarcinoma, micropapillary adenocarcinoma.

migration, and invasion $(14,15)$. Immunohistochemical analysis of gastric cancer tissue confirmed that low DUSP4 expression was associated with sex, tumor size, depth of invasion, and distant metastasis (16).

In this study, we evaluated the relationship between DUSP4 expression and the clinicopathological characteristics of gastric cancer using semi-quantitative immunohistochemical (IHC) staining in a large series of resected gastric cancer samples. Additionally, the association between DUSP4 expression and specific molecular subtypes was evaluated.

\section{Materials and Methods}

Clinical data and pathological evaluation. Gastric cancer cases were retrospectively enrolled as patients who underwent surgical resection between February 2005 and July 2010 at the Hanyang University Hospital. A total of 520 cases were collected, and 9 cases were excluded from the study because they had distant metastasis at the time of diagnosis or neoadjuvant therapy. In addition, two cases had a positive surgical margin, and one case was excluded because the patient died immediately after surgery. Consequently, 508 cases were included in this study. To compare the expression of DUSP4, 27, 54, and 63 samples of the normal gastric mucosa ( $\geq 1$ $\mathrm{cm}$ from the tumor), adenoma, and lymph node metastasis were additionally obtained, respectively.

Medical records were reviewed to confirm the patient's clinical information including age, sex, adjuvant chemotherapy, death, and recurrence date. A review of hematoxylin and eosin (H\&E) slides and pathologic reports was performed by two pathologists (SSB and SSP). Tumor location, and size, gross type, histology, lymphovascular invasion and perineural invasion, and tumor-node-metastasis (TNM) stage were evaluated. Tumor histology was classified based on the Lauren classification and 2019 WHO classification, and the TNM stage was classified according to the $8^{\text {th }}$ edition of the American Joint Committee on Cancer (AJCC). The clinicopathological characteristics of gastric cancer patients are summarized in Table I. 

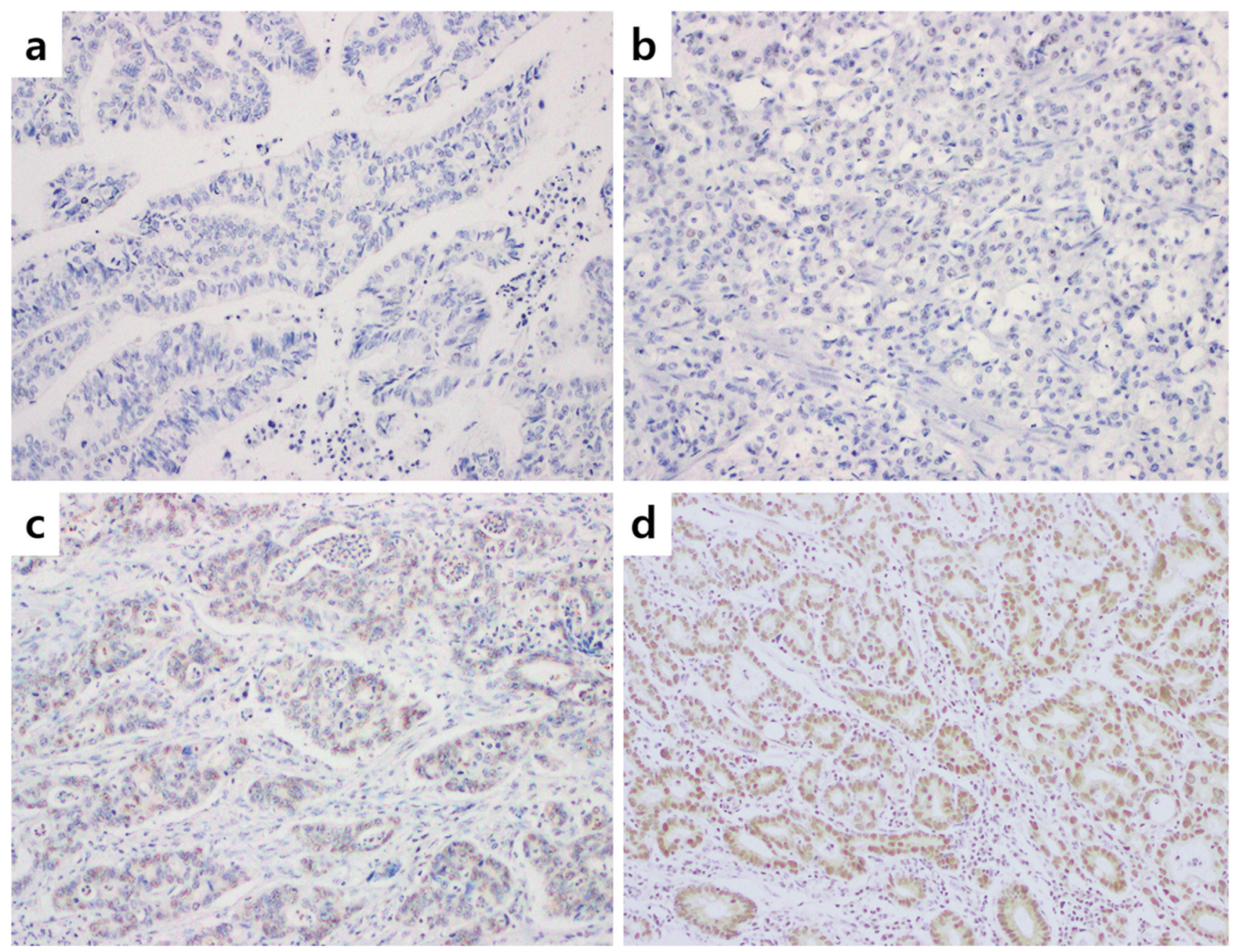

Figure 1. Immunohistochemical staining of dual-specificity protein phosphatase 4 (DUSP4) in gastric cancer. (a): Negative, (b): weakly positive expression, (c): intermediate expression, (d): strong positive expression, $\times 200$.

Tissue microarray construction and immunohistochemistry. Tissue microarray systems (Tissue Microarray Set, Labro, Seoul, Republic of Korea) and formalin-fixed paraffin-embedded (FFPE) tissue were used for tissue microarray (TMA) construction. The blocks of gastrectomy or endoscopic submucosal dissection (ESD) specimens were used for normal gastric mucosa, tubular adenoma, and gastric cancer tissue. For LN tissue, the block with the largest metastatic tumor in the LN dissection specimen was used. After determining the representative area on the $\mathrm{H} \& \mathrm{E}$ slides by light microscopy, tissue was collected through a $3.0 \mathrm{~mm}$ punch.

Immunohistochemistry was performed to evaluate the expression of DUSP4. Deparaffinization and rehydration were performed on 4 $\mu \mathrm{m}$ sections by immersing the sections in xylene and graded series of ethanol. Heat-induced epitope retrieval $\left(100^{\circ} \mathrm{C}\right.$ for $20 \mathrm{~min}$ in sodium citrate buffer) and blocking of endogenous peroxidase using the peroxidase-blocking solution (S2023, Dako, Glostrup, Denmark) for $15 \mathrm{~min}$ were performed. Sections were incubated at $4^{\circ} \mathrm{C}$ overnight with the dilated DUSP4 antibody (1:200, Rabbit DUSP4 polyclonal antibody, ab72593, Abcam, Cambridge, MA, USA).
Table II. DUSP4 expression in various gastric tissues.

\begin{tabular}{lrrrr}
\hline Samples & Cases & \multicolumn{3}{c}{ DUSP4 expression } \\
\cline { 3 - 5 } & & $\begin{array}{c}\text { Low } \\
\text { expression (\%) }\end{array}$ & $\begin{array}{c}\text { High } \\
\text { expression (\%) }\end{array}$ & $p$-Value \\
\hline Normal mucosa & 27 & $4(14.8 \%)$ & $23(85.2 \%)$ & $<0.001$ \\
Tubular adenoma & 54 & $7(13.0 \%)$ & $47(87.0 \%)$ & \\
Gastric cancer & 508 & $233(45.9 \%)$ & $275(54.1 \%)$ & \\
LN metastasis & 63 & $34(54.0 \%)$ & $29(46.0 \%)$ & \\
& & & &
\end{tabular}

DUSP4, Dual-specificity protein phosphatase 4; LN, lymph node.

Labeled polymer and 3,3'-diaminobenzidine tetrahydrochloride chromogen (K5007, EnVision ${ }^{\mathrm{TM}}$ Detection System, Dako, Glostrup, Denmark) were sequentially used for detection according to the manufacturer's instructions. 


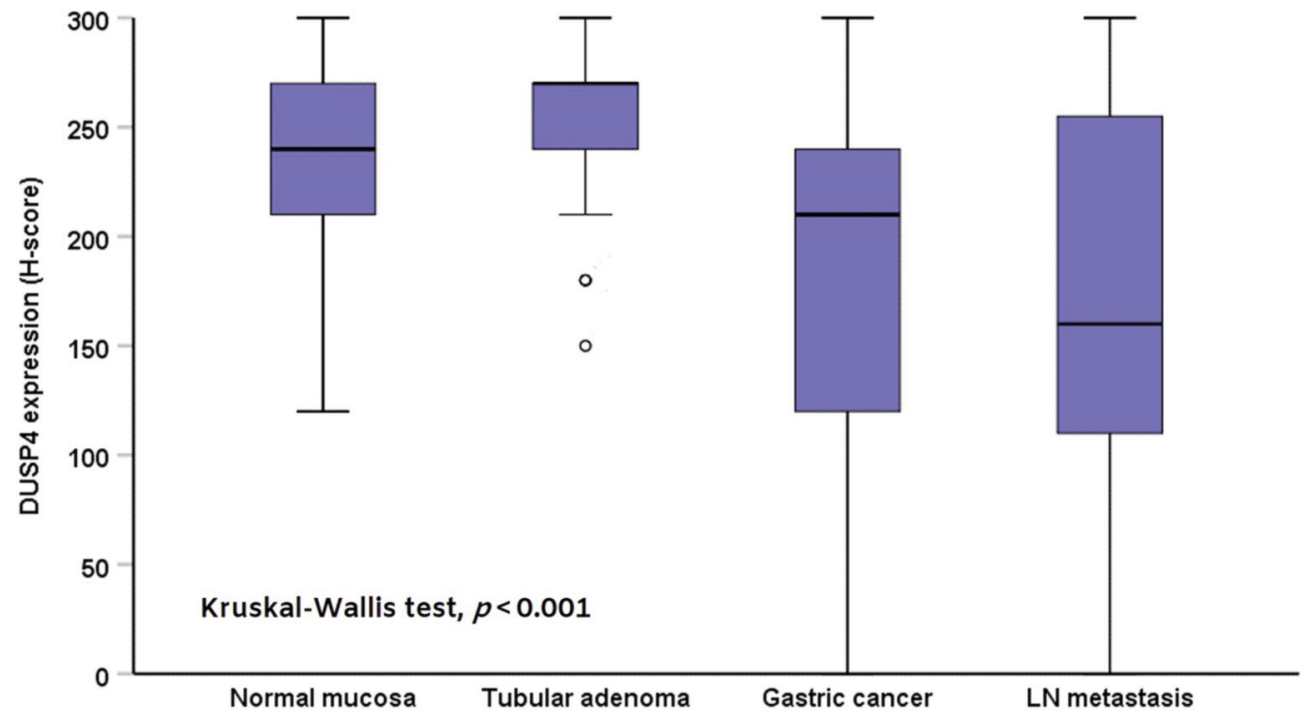

Figure 2. The pattern of Dual-specificity protein phosphatase 4 (DUSP4) expression in normal mucosa, tubular adenoma, gastric cancer, and lymph node (LN) metastasis ( $p<0.001$, Kruskal-Wallis test).

Interpretation of IHC staining. DUSP4 expression was evaluated by two pathologists without access to clinical data (SSB and SSP). For the semi-quantitative assessment, histoscore (H-score) was calculated based on the intensity and percentage of nuclear DUSP4 expression. The intensity of staining was classified as 0 to 3 ( 0 : negative, 1: weak, 2: intermediate, 3: strong) and the positive rate was scored from 0 to 100 . Following this, the optimal cutoff value of DUSP4 expression was determined using the receiver operating characteristics (ROC) curve, and cases were divided into high or low expression.

Microsatellite instability and Epstein-Barr virus status. The TCGA network classified gastric cancer into four molecular subtypes using comprehensive molecular evaluation: Epstein-Barr virus (EBV), microsatellite instability (MSI), genomic stability (GS), and chromosomal instability (CIN) (17). In this study, IHC staining of mismatch repair proteins was performed to identify the MSI subtype and EBV-encoded RNA in situ hybridization (EBER-ISH) was performed to confirm the EBV subtype. IHC staining was performed on the entire tumor section for MLH1 (G168-728, Cell Marque, Rocklin, CA, USA), PMS2 (MRQ-28, Cell Marque, CA, USA), MSH2 (G219-1129, Cell Marque, CA, USA), and MSH6 (PU29, Leica Biosystems, Nussloch, Germany) and classified as MSI subtype when one or more results were negative. Cases with diffuse positivity when stained using the INFORM EBER Probe (Roche, Basel, Switzerland) on TMA slides were classified as EBV subtype.

HER2 status. IHC and silver DNA in situ hybridization (SISH) tests were performed on all sections of the TMA to evaluate HER2 amplification. Automatic staining was performed according to the manufacturer's instructions with a rabbit monoclonal anti-HER-2 antibody (4B5, Roche) and INFORM HER2 Dual ISH DNA probe cocktail (Roche). HER2 amplified cases were defined as an IHC score 3 or a HER2/ chromosome 17 (CEP17) ratio of $\geq 2.0$ (18).
Bioinformatics. Kaplan-Meier plotter (KM plotter, http://kmplot.com/ analysis/) is an online analysis tool that provides the gene expression data for 1,065 gastric cancer samples (19). It is used to predict the association between the mRNA expression of biomarker candidates and the survival of gastric cancer patients. The effect of DUSP4 expression on the prognosis of gastric cancer patients was estimated using this tool in this study.

Statistical analyses. The Kruskal-Wallis test was performed to compare DUSP4 expression in normal gastric mucosa, tubular adenoma, gastric cancer, and lymph node metastasis. Pearson's chisquare $\left(\chi^{2}\right)$ and Fisher's exact tests were performed to evaluate the correlation between DUSP4 expression and clinicopathological factors. The Kaplan-Meier method with log-rank test was performed to evaluate the influence of DUSP4 expression on overall survival (OS) and recurrence-free survival (RFS), and univariate and multivariate Cox regression analyses were performed to identify the significant prognostic factors. SPSS software version 25.0 (IBM, Armonk, USA) was used for all statistical analyses, and $p$-value $<0.05$ was considered statistically significant.

\section{Results}

The pattern of DUSP4 expression. Nuclear DUSP4 expression was variable, and representative images are presented in Figure 1. Cases were divided into highexpression group ( $\mathrm{H}$-score $\geq 200$ ) and low-expression group (H-score <200) using the ROC curve. High DUSP4 expression was observed in 23 cases $(85.2 \%)$ of normal gastric mucosa, 47 cases $(87.0 \%)$ of tubular adenoma, 275 cases $(54.1 \%)$ of gastric cancer, and 29 cases $(46.0 \%)$ of LN tissue with gastric cancer metastasis (Table II). DUSP4 
Table III. Correlation between DUSP4 expression and clinicopathological features and molecular characteristics $(n=508)$.

\begin{tabular}{|c|c|c|c|}
\hline \multirow[t]{2}{*}{ Variables } & \multicolumn{2}{|c|}{ DUSP4 expression } & \multirow[t]{2}{*}{$p$-Value } \\
\hline & $\begin{array}{c}\text { Low } \\
\text { expression }(\%)\end{array}$ & $\begin{array}{c}\text { High } \\
\text { expression }(\%)\end{array}$ & \\
\hline \multicolumn{3}{|l|}{ Age } & 0.984 \\
\hline$<65$ years & $140(45.9 \%)$ & $165(54.1 \%)$ & \\
\hline$\geq 65$ years & $93(45.8 \%)$ & $110(54.2 \%)$ & \\
\hline \multicolumn{3}{|l|}{ Gender } & 0.284 \\
\hline Female & $66(42.3 \%)$ & $90(57.7 \%)$ & \\
\hline Male & $167(47.4 \%)$ & $185(52.6 \%)$ & \\
\hline \multicolumn{3}{|l|}{ Tumor size } & $<0.001$ \\
\hline$<4.0 \mathrm{~cm}$ & $98(35.0 \%)$ & $182(65.0 \%)$ & \\
\hline$\geq 4.0 \mathrm{~cm}$ & $135(59.2 \%)$ & $93(40.8 \%)$ & \\
\hline \multicolumn{3}{|l|}{ Location } & 0.575 \\
\hline Proximal & $80(44.2 \%)$ & $101(55.8 \%)$ & \\
\hline Distal & $153(46.8 \%)$ & $174(53.2 \%)$ & \\
\hline \multicolumn{3}{|l|}{ Histologic type* } & 0.252 \\
\hline Differentiated & $85(42.7 \%)$ & $114(57.3 \%)$ & \\
\hline $\begin{array}{l}\text { Undifferentiated } \\
\text { and others }\end{array}$ & $148(47.9 \%)$ & $161(52.1 \%)$ & \\
\hline \multicolumn{3}{|l|}{ Lauren } & 0.065 \\
\hline Intestinal & $87(41.0 \%)$ & $125(59.0 \%)$ & \\
\hline $\begin{array}{l}\text { Diffuse and } \\
\text { mixed }\end{array}$ & $146(49.3 \%)$ & $150(50.7 \%)$ & \\
\hline \multicolumn{3}{|l|}{ pT category } & $<0.001$ \\
\hline pT1 and pT2 & $108(34.3 \%)$ & $207(65.7 \%)$ & \\
\hline pT3 and pT4 & $125(64.8 \%)$ & $68(35.2 \%)$ & \\
\hline \multicolumn{3}{|l|}{ Nodal status } & $<0.001$ \\
\hline Negative & $115(38.5 \%)$ & $184(61.5 \%)$ & \\
\hline Positive & $118(56.5 \%)$ & $91(43.5 \%)$ & \\
\hline \multicolumn{3}{|l|}{ Stage } & $<0.001$ \\
\hline I & $93(33.1 \%)$ & $188(66.9 \%)$ & \\
\hline II and III & $140(61.7 \%)$ & $87(38.3 \%)$ & \\
\hline \multicolumn{3}{|l|}{$\begin{array}{l}\text { Lymphovascular } \\
\text { invasion }\end{array}$} & $<0.001$ \\
\hline Absent & $94(37.0 \%)$ & $160(63.0 \%)$ & \\
\hline Present & $139(54.7 \%)$ & $115(45.3 \%)$ & \\
\hline \multicolumn{3}{|l|}{$\begin{array}{l}\text { Perineural } \\
\text { invasion }\end{array}$} & $<0.001$ \\
\hline Absent & $110(34.9 \%)$ & $205(65.1 \%)$ & \\
\hline Present & $123(63.7 \%)$ & $70(36.3 \%)$ & \\
\hline \multicolumn{4}{|l|}{ EBV status } \\
\hline Negative & $213(44.9 \%)$ & $261(55.1 \%)$ & 0.116 \\
\hline & $20(58.8 \%)$ & $14(41.2 \%)$ & \\
\hline \multicolumn{4}{|l|}{ MSI status } \\
\hline MSS & $219(53.2 \%)$ & $249(46.8 \%)$ & 0.151 \\
\hline MSI & $14(35.0 \%)$ & $26(65.0 \%)$ & \\
\hline \multicolumn{4}{|l|}{ HER2 status } \\
\hline No amplification & $220(45.5 \%)$ & $264(54.5 \%)$ & 0.403 \\
\hline Amplification & $13(54.2 \%)$ & $11(45.8 \%)$ & \\
\hline
\end{tabular}

*Differentiated: Well-differentiated tubular adenocarcinoma, moderately differentiated tubular adenocarcinoma, papillary adenocarcinoma; Undifferentiated: poorly differentiated tubular adenocarcinoma, poorly cohesive carcinoma; Others: mucinous adenocarcinoma, other histologic subtypes; Stage, AJCC $8^{\text {th }}$ edition. EBV, Epstein-Barr virus; MSS, microsatellite stable; MSI, microsatellite instability; HER2, human epidermal growth factor receptor-2. expression was significantly lower in gastric cancer and lymph node metastasis than in normal gastric mucosa and tubular adenoma ( $p<0.001$, Kruskal-Wallis test) (Figure 2). There was no significant difference in DUSP4 expression between normal gastric mucosa and tubular adenoma and between gastric cancer and lymph node metastasis.

The correlation between DUSP4 expression and clinicopathological features. The correlation between DUSP4 expression and clinicopathological features was investigated and is summarized in Table III. Low expression of DUSP4 was significantly correlated with larger tumor size $(p<0.001)$, presence of lymphovascular invasion $(p<0.001)$, presence of perineural invasion $(p<0.001)$, higher $\mathrm{pT}$ category $(p<0.001)$, nodal metastasis $(p<0.001)$ and higher stage $(p<0.001)$. No significant correlation was observed between DUSP4 expression and other features including age, gender, tumor location, Lauren classification, and histological type.

The correlation between DUSP4 expression and molecular characteristics. According to the TCGA molecular classification, 34 cases $(6.7 \%)$ were classified as EBV subtype and 40 cases $(7.9 \%)$ were classified as MSI subtype. The remaining 434 cases $(85.4 \%)$ were classified as GS or CIN subtype. In this study, these two subtypes were not distinguished and classified as EBV-negative or microsatellite stable (MSS). There were 24 HER2-amplified cases $(4.7 \%)$ and these cases were EBV-negative or MSS. There was no correlation between DUSP4 expression and molecular characteristics (Table III).

Prognostic significance of various factors including DUSP4 expression. In univariate analyses, various factors showed a significant association with OS or RFS. Low DUSP4 expression $(p=0.028)$, older age $(p<0.001)$, larger tumor size $(p<0.001)$, diffuse and mixed type of Lauren classification $(p=0.016)$, higher $\mathrm{pT}$ category $(p<0.001)$, nodal metastasis $(p<0.001)$, higher TNM stage $(p<0.001)$, lymphovascular invasion $(p<0.001)$, and perineural invasion $(p<0.001)$ were associated with short OS. Low DUSP4 expression $(p=0.004)$, larger tumor size $(p<0.001)$, undifferentiated and other types of WHO histological classification $(p<0.001)$, diffuse and mixed type of Lauren classification $(p<0.001)$, higher $\mathrm{pT}$ category $(p<0.001)$, nodal metastasis $(p<0.001)$, higher TNM stage $(p<0.001)$, lymphovascular invasion $(p<0.001)$, and perineural invasion $(p<0.001)$ were associated with short RFS. In multivariate analyses, older age $(p<0.001)$, higher TNM stage $(p<0.001)$, and perineural invasion $(p=0.041)$ were associated with short OS, and higher TNM stage $(p<0.001)$ was associated with short RFS. However, low DUSP4 expression was not statistically significant (Table IV). Kaplan-Meier survival analyses revealed that low DUSP4 expression was significantly associated with short OS and RFS (log-rank test, $p=0.027$ and $p=0.003$, respectively; Figure 3 ). Similar to our results, the 
in vivo $35: 131-140(2021)$

Table IV. Univariate and multivariate Cox regression analyses for overall survival and recurrence-free survival ( $n=508)$.

\begin{tabular}{|c|c|c|c|c|c|c|}
\hline \multirow[b]{3}{*}{ Variables } & \multicolumn{6}{|c|}{ Overall survival } \\
\hline & \multicolumn{3}{|c|}{ Univariate analysis } & \multicolumn{3}{|c|}{ Multivariate analysis } \\
\hline & HR & $95 \% \mathrm{CI}$ & $p$-Value & HR & $95 \% \mathrm{CI}$ & $p$-Value \\
\hline $\begin{array}{l}\text { DUSP4 expression } \\
\text { (high } v s . \text { low) }\end{array}$ & 1.395 & $1.035-1.878$ & 0.028 & 1.111 & $0.811-1.523$ & 0.512 \\
\hline $\begin{array}{l}\text { Age group } \\
(<65 v s . \geq 65)\end{array}$ & 1.827 & $1.357-2.460$ & $<0.001$ & 1.954 & $1.446-2.641$ & $<0.001$ \\
\hline $\begin{array}{l}\text { Tumor size } \\
(<4.0 \mathrm{~cm} v s . \geq 4.0 \mathrm{~cm})\end{array}$ & 2.308 & $1.701-3.130$ & $<0.001$ & 1.080 & $0.744-1.568$ & 0.686 \\
\hline Histologic type & 1.314 & $0.962-1.794$ & 0.086 & & & \\
\hline Lauren classification & 1.465 & $1.074-1.999$ & 0.016 & 1.001 & $0.714-1.403$ & 0.996 \\
\hline $\begin{array}{l}\text { pT category } \\
(\mathrm{T} 1-2 \text { vs. T3-4) }\end{array}$ & 3.581 & $2.635-4.866$ & $<0.001$ & & & \\
\hline $\begin{array}{l}\text { Nodal status } \\
\text { (negative } v s \text {. positive) }\end{array}$ & 3.361 & $2.467-4.579$ & $<0.001$ & & & \\
\hline Stage (I vs. II, III) & 3.671 & $2.663-5.059$ & $<0.001$ & 2.635 & $1.530-4.539$ & $<0.001$ \\
\hline LVI (absent $v s$. present) & 2.786 & $2.023-3.838$ & $<0.001$ & 1.007 & $0.617-1.642$ & 0.978 \\
\hline \multirow[t]{3}{*}{ PNI (absent $v s$. present) } & 3.211 & $2.370-4.348$ & $<0.001$ & 1.616 & $1.021-2.557$ & 0.041 \\
\hline & \multicolumn{6}{|c|}{ Recurrence-free survival } \\
\hline & \multicolumn{3}{|c|}{ Univariate analysis } & \multicolumn{3}{|c|}{ Multivariate analysis } \\
\hline Variables & HR & $95 \% \mathrm{CI}$ & $p$-Value & HR & $95 \% \mathrm{CI}$ & $p$-Value \\
\hline $\begin{array}{l}\text { DUSP4 expression } \\
\text { (high } v s . \text { low) }\end{array}$ & 1.776 & $1.202-2.623$ & 0.004 & 1.076 & $0.719-1.610$ & 0.721 \\
\hline $\begin{array}{l}\text { Age group } \\
(<65 v s . \geq 65)\end{array}$ & 0.949 & $0.638-1.412$ & 0.796 & & & \\
\hline $\begin{array}{l}\text { Tumor size } \\
(<4.0 \mathrm{~cm} v s . \geq 4.0 \mathrm{~cm})\end{array}$ & 4.777 & $3.026-7.542$ & $<0.001$ & 1.335 & $0.803-2.220$ & 0.266 \\
\hline Histologic type & 2.964 & $1.820-4.828$ & $<0.001$ & & & \\
\hline Lauren classification & 3.584 & $2.179-5.895$ & $<0.001$ & 1.620 & $0.964-2.721$ & 0.068 \\
\hline $\begin{array}{l}\text { pT category } \\
\text { (T1-2 vs. T3-4) }\end{array}$ & 10.091 & $6.065-16.789$ & $<0.001$ & & & \\
\hline $\begin{array}{l}\text { Nodal status } \\
\text { (negative } v s \text {. positive) }\end{array}$ & 9.511 & $5.651-16.007$ & $<0.001$ & & & \\
\hline Stage (I vs. II, III) & 13.636 & $7.294-25.494$ & $<0.001$ & 5.258 & $2.169-12.744$ & $<0.001$ \\
\hline LVI (absent $v s$. present) & 9.461 & $5.181-17.275$ & $<0.001$ & 1.777 & $0.789-4.000$ & 0.165 \\
\hline PNI (absent $v s$. present) & 7.322 & $4.604-11.644$ & $<0.001$ & 1.430 & $0.789-2.592$ & 0.238 \\
\hline
\end{tabular}

HR, Hazard ratio; 95\% CI, 95\% confidence interval; Histologic type, differentiated vs. undifferentiated and others; Lauren classification, intestinal $v s$. diffuse and mixed; Stage, AJCC $8^{\text {th }}$ edition; LVI, lymphovascular invasion; PNI, perineural invasion.

analysis using the KM plotter demonstrated that low mRNA expression of DUSP4 was associated with unfavorable survival outcomes (log-rank test, $p<0.001$; Figure 4).

\section{Discussion}

In the present study, immunohistochemical staining for DUSP4 was performed in normal gastric mucosa, tubular adenoma, gastric cancer, and LN tissue with gastric cancer metastasis. Our results demonstrated that the expression of
DUSP4 was significantly lower in gastric cancer and metastatic tumors than that in normal gastric mucosa and tubular adenoma. Low expression of DUSP4 was significantly associated with larger tumor size, higher pT stage, nodal metastasis, lymphovascular invasion, and perineural invasion, which suggests that DUSP4 down-regulation is associated with gastric cancer progression. Similar to the KM plotter analysis results, Kaplan-Meier survival analyses revealed that low DUSP4 expression was significantly associated with overall survival and recurrence-free survival. 

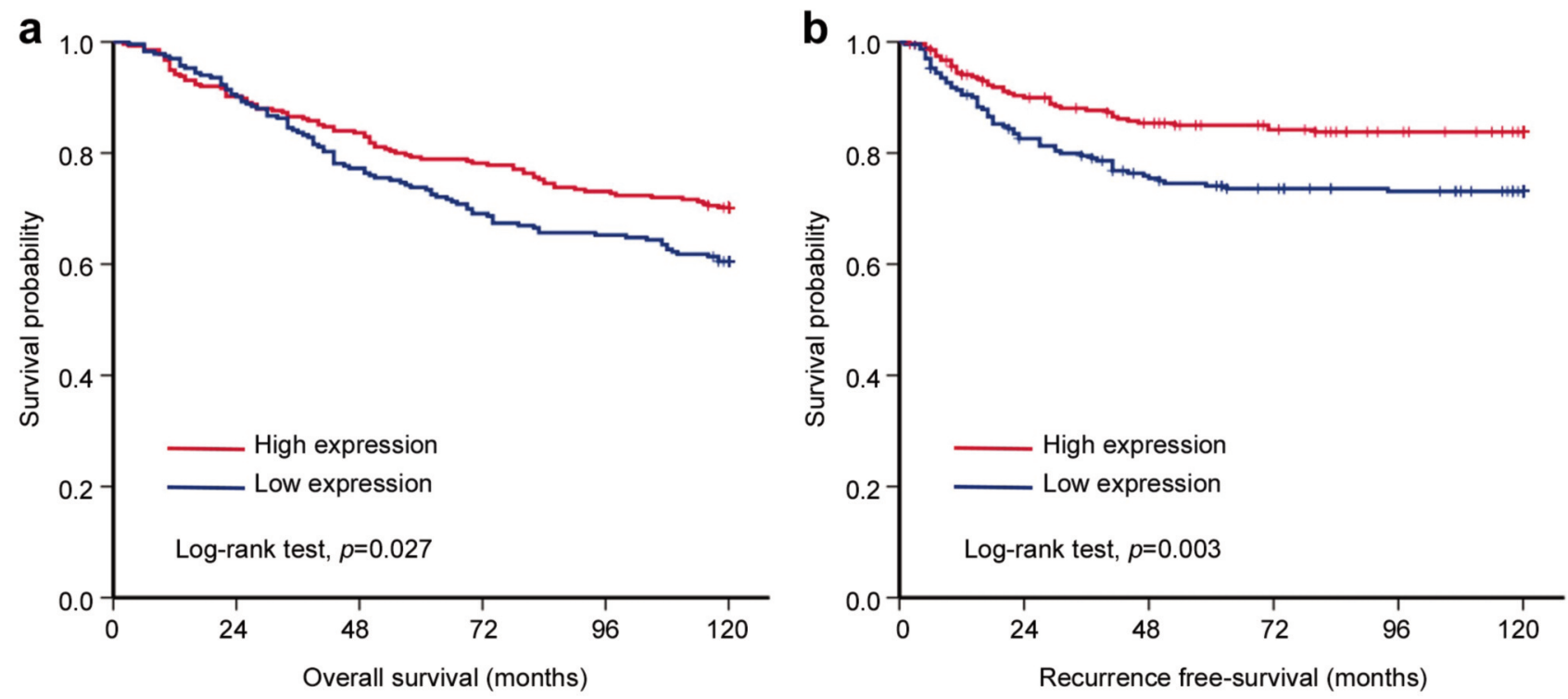

Figure 3. Kaplan-Meier survival analyses. (a) Overall survival was significantly shorter in cases with low expression than in cases with high expression (Log-rank test, $p=0.027$ ). (b) Recurrence-free survival (RFS) was significantly shorter in cases with low expression than in cases with high expression (Log-rank test, $p=0.003$ ).

DUSPs are a subclass of protein tyrosine phosphatases that dephosphorylate threonine and tyrosine residues on MAPKs and consequently act as negative regulators of MAPK signaling. DUSP4 is included in the first subfamily together with DUSP1, DUSP2, and DUSP5. It is mainly located in the nucleus and is known to show substrate specificity for MAP kinases p38, c-Jun N-terminal kinase (JNK), and extracellular-signal-regulated kinase (ERK) (20). Promoter hypermethylation and copy loss are representative molecular alterations affecting DUSP4 expression in cancer. Waha et al. demonstrated de novo methylation and transcriptional silencing of DUSP4/MKP-2 in primary glioma tissues and glioma cell lines (21) and Schmid et al. reported that DUSP4 promoter hypermethylation repressed DUSP4 expression in diffuse large B-cell lymphoma (9). Chitale et al. and Mazumdar et al. revealed that copy loss of DUSP4 at chromosome 8p was found in EGFR-mutant lung cancers and estrogen receptor (ER)-negative breast cancers $(22,23)$. Several studies have shown that the function, whether a tumor suppressor or tumor promoter, varies depending on the type of cancer, and the exact impact of DUSP4 expression on cancer progression and prognosis is still unclear.

Recently, Baglia et al. and Gaggianesi et al. reported that low expression of DUSP4 is generally considered to be associated with a poor prognosis or aggressive features in breast cancer $(12,24)$. Mazumdar et al. demonstrated that the overexpression of DUSP4 suppressed the MAPK, NF$\mathrm{KB}$, and $\mathrm{Rb}$ signaling pathways and inhibited cell growth
(23). Balko et al. showed that low DUSP4 expression was correlated with high ERK activity and basal-like subtype. DUSP4 promoter methylation is most common in basal-like subtype among the molecular subtypes of breast cancer (25). Ichimanda et al. reported that colorectal cancer tissue showed a higher expression of DUSP4 than that in normal tissue. However, compared to the superficial region, DUSP4 expression decreased in the deep region of the tumor, and the activity of ERKs increased (26). Saigusa et al. demonstrated that decreased DUSP4 expression in colorectal cancer was significantly correlated with tumor progression and distance metastasis (10). Chitale et al. showed that $8 \mathrm{p}$ loss was observed more frequently in EGFR mutant adenocarcinoma of the lung, and re-expression of DUSP4 reduced growth and knockdown of DUSP4 leads to enhanced growth (22). They suggested that DUSP4 might act as a tumor suppressor in human malignancy.

Some authors have reported that DUSP4 in tumor carcinogenesis may play a role as a tumor promoter. Briston et al. reported that ERK1/2 activity was increased when DUSP4 protein was highly expressed and that depletion of DUSP4 decreased ERK activity in EGFR-mutant lung cancer cells (5). Lee et al. demonstrated that DUSP4 expression was increased in thyroid cancer cell lines (TPC1, WRO82-1, and XTC) (6). Ma et al. showed that high DUSP4 expression was associated with aggressive features such as lymph node metastasis and extrathyroidal extension in papillary thyroid carcinoma (7). Kim et al. reported that high DUSP4 expression was associated with poor prognosis in early $\mathrm{T}$ - 


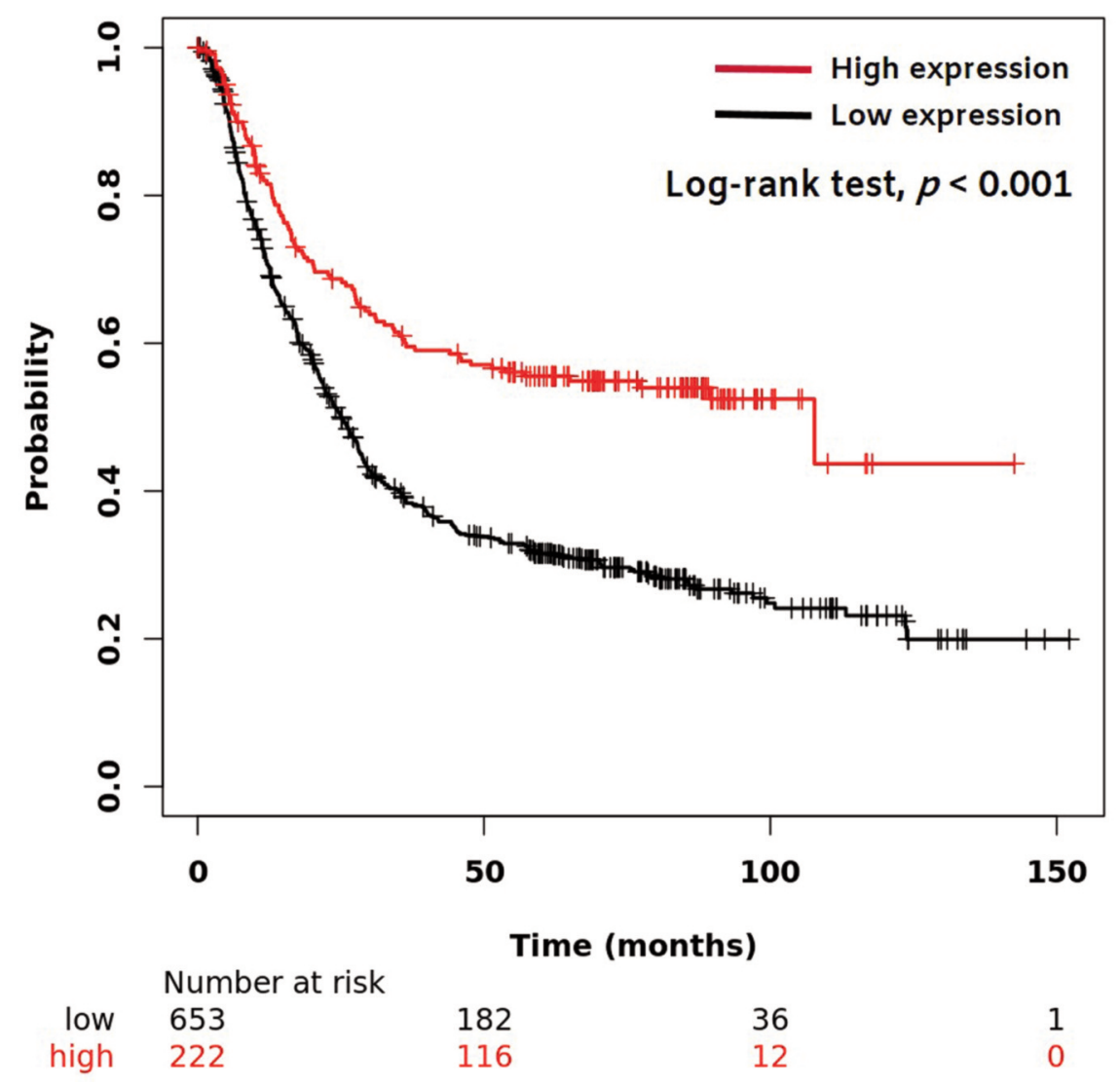

Figure 4. Analysis using the Kaplan-Meier (KM) plotter demonstrated that low mRNA expression of DUSP4 was associated with unfavorable survival outcome ( $n=875 ;$ Log-rank test, $p<0.001)$.

stage breast cancer (13). Gröschl et al. showed that DUSP4 is associated with microsatellite instability (MSI) in colorectal cancer and DUSP4 overexpression increased cell proliferation in colorectal cancer cell lines (27). They suggested that DUSP4 might act as a tumor promoter in human malignancy.

In gastric cancer, there is no conclusive report regarding the exact role of DUSP4 expression in tumor progression and prognosis. Kang et al. reported that DUSP4 overexpression increased doxorubicin resistance in gastric cancer cells and the knockdown of DUSP4 increased the cytotoxicity of gastric cancer cells to doxorubicin. Up-regulation of DUSP4 promotes the epithelial-mesenchymal transition (14). Xu et al. revealed that the knockdown of DUSP4 reduced the mobility and invasiveness of gastric cancer cells, and DUSP4 mRNA expression was increased in gastric carcinoma compared to that in the adjacent tissues. DUSP4 expression showed a significant increase in 30 gastric cancer tissues detected by immunohistochemistry and western blotting (15). Zhang et al. demonstrated that low DUSP4 expression was significantly associated with various factors related to cancer progression, such as larger tumor size, deep invasion of the tumor, and distant metastasis in a study of 89 patients with gastric cancer (16). In our study, low DUSP4 expression was significantly correlated with aggressive phenotypes and poor prognosis in a large series of 508 patients with gastric cancer. The association between DUSP4 expression and specific molecular subtypes was investigated, however, there was no significant correlation between DUSP4 expression and specific molecular subtypes.

As a strength of this study, it was possible to evaluate the clinicopathological significance of DUSP4 expression in gastric cancer through larger number of cases than previous studies, and similar results were confirmed using bioinformatics analysis. Also, this is the first study to investigate the association between the expression of DUSP4 and a specific molecular subtype of gastric cancer. However, there is a limitation in that we only evaluated the expression of DUSP4 through immunohistochemistry. The molecular mechanism underlying the role of DUSP4 expression in gastric cancer was not studied, and further studies such as DUSP4 promoter hypermethylation are needed. 
In conclusion, our results showed that DUSP4 expression in gastric cancer and lymph node metastasis was reduced compared to that in normal tissue and tubular adenoma. Low DUSP4 expression is associated with cancer progression and poor prognosis in patients with gastric cancer. Our results suggest that DUSP4 may act as a tumor suppressor in gastric cancer. The exact role of DUSP4 in gastric cancer and its potential as a novel therapeutic target for gastric cancer should be investigated in further studies.

\section{Compliance with Ethical Standards}

The Institutional Review Board of Hanyang University Hospital (HYUH 2019-04-032-001) approved the study and waived the requirement for informed consent.

\section{Conflicts of Interest}

The Authors declare that no potential conflicts of interest exist relevant to this article.

\section{Authors' Contributions}

Seongsik Bang: tissue microarray construction; assessment of the immunohistochemical slides, review of histological material, data analysis, drafting of the manuscript, preparation of the illustrations, preparation of the final text. Seungyun Jee: review of histological material; Hyunsung Kim: review of histological material; Kiseok Jang: revision of the manuscript; Hosub Park: revision of the manuscript; Jaekyung Myung: revision of the manuscript; Dongho Choi: provided clinical data and antibody; Su-Jin Shin: review of histological material, choice of cases for the study, tissue microarray construction; Seung Sam Paik: elaboration of the research concept, general supervision of the study, revision of the manuscript and preparation of the final text.

\section{Acknowledgements}

This research was supported by Grants from the Medical Research Center (2017R1A5A2015395) and funded by the National Research Foundation (NRF) of the Ministry of Education, Science and Technology (MEST).

\section{References}

1 Bray F, Ferlay J, Soerjomataram I, Siegel RL, Torre LA and Jemal A: Global cancer statistics 2018: Globocan estimates of incidence and mortality worldwide for 36 cancers in 185 countries. CA Cancer J Clin 68(6): 394-424, 2018. PMID: 30207593. DOI: $10.3322 / \mathrm{caac} .21492$

2 Collaborators GBDSC: The global, regional, and national burden of stomach cancer in 195 countries, 1990-2017: A systematic analysis for the global burden of disease study 2017. Lancet Gastroenterol Hepatol 5(1): 42-54, 2020. PMID: 31648970. DOI: $10.1016 /$ S2468-1253(19)30328-0

3 Pellino A, Riello E, Nappo F, Brignola S, Murgioni S, Djaballah SA, Lonardi S, Zagonel V, Rugge M, Loupakis F and Fassan M:
Targeted therapies in metastatic gastric cancer: Current knowledge and future perspectives. World J Gastroenterol 25(38): 5773-5788, 2019. PMID: 31636471. DOI: 10.3748/ wjg.v25.i38.5773

4 Seternes OM, Kidger AM and Keyse SM: Dual-specificity map kinase phosphatases in health and disease. Biochim Biophys Acta Mol Cell Res 1866(1): 124-143, 2019. PMID: 30401534. DOI: 10.1016/j.bbamcr.2018.09.002

5 Britson JS, Barton F, Balko JM and Black EP: Deregulation of dusp activity in egfr-mutant lung cancer cell lines contributes to sustained erk1/2 signaling. Biochem Biophys Res Commun 390(3): 849-854, 2009. PMID: 19836351. DOI: 10.1016/j.bbrc. 2009.10.061

6 Lee EK, Chung KW, Yang SK, Park MJ, Min HS, Kim SW and Kang HS: DNA methylation of mapk signal-inhibiting genes in papillary thyroid carcinoma. Anticancer Res 33(11): 4833-4839, 2013. PMID: 24222120.

7 Ma B, Shi R, Yang S, Zhou L, Qu N, Liao T, Wang Y, Wang Y and Ji Q: Dusp4/mkp2 overexpression is associated with braf(v600e) mutation and aggressive behavior of papillary thyroid cancer. Onco Targets Ther 9: 2255-2263, 2016. PMID: 27143921. DOI: $10.2147 / O T T . S 103554$

8 Yip-Schneider MT, Lin A and Marshall MS: Pancreatic tumor cells with mutant k-ras suppress erk activity by mek-dependent induction of map kinase phosphatase-2. Biochem Biophys Res Commun 280(4): 992-997, 2001. PMID: 11162624. DOI: $10.1006 /$ bbrc. 2001.4243

9 Schmid CA, Robinson MD, Scheifinger NA, Muller S, Cogliatti S, Tzankov A and Muller A: Dusp4 deficiency caused by promoter hypermethylation drives jnk signaling and tumor cell survival in diffuse large b cell lymphoma. J Exp Med 212(5): 775-792, 2015. PMID: 25847947. DOI: 10.1084/jem.20141957

10 Saigusa S, Inoue Y, Tanaka K, Toiyama Y, Okugawa Y, Shimura T, Hiro J, Uchida K, Mohri Y and Kusunoki M: Decreased expression of dusp4 is associated with liver and lung metastases in colorectal cancer. Med Oncol 30(3): 620, 2013. PMID: 23749251. DOI: 10.1007/s12032-013-0620-x

11 Menyhart O, Kakisaka T, Pongor LS, Uetake H, Goel A and Gyorffy B: Uncovering potential therapeutic targets in colorectal cancer by deciphering mutational status and expression of druggable oncogenes. Cancers (Basel) 11(7), 2019. PMID: 31337155. DOI: $10.3390 /$ cancers 11070983

12 Baglia ML, Cai Q, Zheng Y, Wu J, Su Y, Ye F, Bao PP, Cai H, Zhao Z, Balko J, Zheng W, Lu W and Shu XO: Dual specificity phosphatase 4 gene expression in association with triple-negative breast cancer outcome. Breast Cancer Res Treat 148(1): 211-220, 2014. PMID: 25281216. DOI: 10.1007/s10549-014-3127-z

13 Kim H, Jang SM, Ahn H, Sim J, Yi K, Chung Y, Han H, Rehman A, Chung MS, Jang K and Paik SS: Clinicopathological significance of dual-specificity protein phosphatase 4 expression in invasive ductal carcinoma of the breast. J Breast Cancer 18(1): 1-7, 2015. PMID: 25834604. DOI: 10.4048/jbc. 2015.18.1.1

14 Kang X, Li M, Zhu H, Lu X, Miao J, Du S, Xia X and Guan W: Dusp4 promotes doxorubicin resistance in gastric cancer through epithelial-mesenchymal transition. Oncotarget 8(55): 9402894039, 2017. PMID: 29212207. DOI: 10.18632/oncotarget. 21522

$15 \mathrm{Xu} \mathrm{X,} \mathrm{Gao} \mathrm{F,} \mathrm{Wang} \mathrm{J,} \mathrm{Tao} \mathrm{L,} \mathrm{Ye} \mathrm{J,} \mathrm{Ding} \mathrm{L,} \mathrm{Ji} \mathrm{W} \mathrm{and} \mathrm{Chen} \mathrm{X:}$ Mir-122-5p inhibits cell migration and invasion in gastric cancer 
by down-regulating dusp4. Cancer Biol Ther 19(5): 427-435, 2018. PMID: 29509059. DOI: 10.1080/15384047.2018.1423925

16 Zhang R, Wang G, Zhang PF, Zhang J, Huang YX, Lu YM, Da W, Sun Q and Zhu JS: Sanguinarine inhibits growth and invasion of gastric cancer cells via regulation of the dusp4/erk pathway. J Cell Mol Med 21(6): 1117-1127, 2017. PMID: 27957827. DOI: $10.1111 / \mathrm{jcmm} .13043$

17 Cancer Genome Atlas Research N: Comprehensive molecular characterization of gastric adenocarcinoma. Nature 513(7517): 202-209, 2014. PMID: 33086305. DOI: 10.1038/nature13480

18 Ruschoff J, Hanna W, Bilous M, Hofmann M, Osamura RY, Penault-Llorca F, van de Vijver M and Viale G: Her2 testing in gastric cancer: A practical approach. Mod Pathol 25(5): 637-650, 2012. PMID: 22222640. DOI: 10.1038/modpathol.2011.198

19 Szasz AM, Lanczky A, Nagy A, Forster S, Hark K, Green JE, Boussioutas A, Busuttil R, Szabo A and Gyorffy B: Crossvalidation of survival associated biomarkers in gastric cancer using transcriptomic data of 1,065 patients. Oncotarget 7(31): 49322-49333, 2016. PMID: 27384994. DOI: 10.18632/ oncotarget.10337

20 Jeffrey KL, Camps M, Rommel C and Mackay CR: Targeting dual-specificity phosphatases: Manipulating map kinase signalling and immune responses. Nat Rev Drug Discov 6(5): 391-403, 2007. PMID: 17473844. DOI: $10.1038 / \mathrm{nrd} 2289$

21 Waha A, Felsberg J, Hartmann W, von dem Knesebeck A, Mikeska T, Joos S, Wolter M, Koch A, Yan PS, Endl E, Wiestler OD, Reifenberger G, Pietsch $T$ and Waha A: Epigenetic downregulation of mitogen-activated protein kinase phosphatase mkp-2 relieves its growth suppressive activity in glioma cells. Cancer Res 70(4): 1689-1699, 2010. PMID: 20124482. DOI: 10.1158/0008-5472.CAN-09-3218

22 Chitale D, Gong Y, Taylor BS, Broderick S, Brennan C, Somwar R, Golas B, Wang L, Motoi N, Szoke J, Reinersman JM, Major J, Sander C, Seshan VE, Zakowski MF, Rusch V, Pao W, Gerald $\mathrm{W}$ and Ladanyi M: An integrated genomic analysis of lung cancer reveals loss of dusp4 in egfr-mutant tumors. Oncogene 28(31): 2773-2783, 2009. PMID: 19525976. DOI: 10.1038/ onc. 2009.135
23 Mazumdar A, Poage GM, Shepherd J, Tsimelzon A, Hartman ZC, Den Hollander P, Hill J, Zhang Y, Chang J, Hilsenbeck SG, Fuqua S, Kent Osborne C, Mills GB and Brown PH: Analysis of phosphatases in er-negative breast cancers identifies dusp4 as a critical regulator of growth and invasion. Breast Cancer Res Treat 158(3): 441-454, 2016. PMID: 27393618. DOI: 10.1007/s 10549-016-3892-y

24 Gaggianesi M, Turdo A, Chinnici A, Lipari E, Apuzzo T, Benfante A, Sperduti I, Di Franco S, Meraviglia S, Lo Presti E, Dieli F, Caputo V, Militello G, Vieni S, Stassi G and Todaro M: Il4 primes the dynamics of breast cancer progression via dusp4 inhibition. Cancer Res 77(12): 3268-3279, 2017. PMID: 28400477. DOI: $10.1158 / 0008-5472$.CAN-16-3126

25 Balko JM, Cook RS, Vaught DB, Kuba MG, Miller TW, Bhola NE, Sanders ME, Granja-Ingram NM, Smith JJ, Meszoely IM, Salter J, Dowsett M, Stemke-Hale K, Gonzalez-Angulo AM, Mills GB, Pinto JA, Gomez HL and Arteaga CL: Profiling of residual breast cancers after neoadjuvant chemotherapy identifies dusp4 deficiency as a mechanism of drug resistance. Nat Med 18(7): 1052-1059, 2012. PMID: 22683778. DOI: 10.1038/ $\mathrm{nm} .2795$

26 Ichimanda M, Hijiya N, Tsukamoto Y, Uchida T, Nakada C, Akagi T, Etoh T, Iha H, Inomata M, Takekawa M and Moriyama M: Downregulation of dual-specificity phosphatase 4 enhances cell proliferation and invasiveness in colorectal carcinomas. Cancer Sci 109(1): 250-258, 2018. PMID: 29150975. DOI: $10.1111 /$ cas. 13444

27 Groschl B, Bettstetter M, Giedl C, Woenckhaus M, Edmonston T, Hofstadter F and Dietmaier W: Expression of the map kinase phosphatase dusp4 is associated with microsatellite instability in colorectal cancer (crc) and causes increased cell proliferation. Int J Cancer 132(7): 1537-1546, 2013. PMID: 22965873. DOI: $10.1002 / \mathrm{ijc} .27834$
Received October 7, 2020

Revised October 23, 2020

Accepted October 26, 2020 